\title{
ADAPTACIONES CURRICULARES PARA LA INCLUSIÓN EDUCATIVA EN LA UNIDAD EDUCATIVA BENJAMIN ARAUJO
}

\author{
Lcda. Nancy Guadalupe Morales Guevara ${ }^{1}$ M.Sc. Velasteguí López Luis Efraín ${ }^{2}$ Lcdo. Pablo Velasteguí López ${ }^{3}$
}

\begin{abstract}
The defense of human rights and democratic values of coexistence and recognition of the diversity of human beings and respect for their differences, the pursuit of equal educational opportunities for all people mutual support, cooperation and exchange of experiences among countries in the field of education for people with special needs is the concern of Latin American countries. Inclusion is a movement to transform education systems to meet the diversity of students is essential to realizing the right to education with equal opportunity of access, retention and integration of all students, with an emphasis on those who are excluded or at risk of being marginalized. UNESCO carries out activities of technical advice, promoting inclusive policies and practices; development and dissemination of publications, research and studies of inclusive education in the region, these actions promote changes in educational practices, eliminating barriers developing quality education for all. Inclusion is a global phenomenon that aims to eliminate the relegation having individuals for their physical needs and intellectual in our country strategies to include in all areas of human activity for people with disabilities develop. Ecuador begins with the inclusion as a state policy with emphasis on inclusive education to address diversity, seeking warmth and quality education, aiming to give everyone a life with dignity, with equal opportunities, that lead to success inclusion of all students with various disabilities, including processes are factors that arouse respect for diversity and recognize the constitutional rights that must be met to people with disabilities. The curricular changes are adjustments or modifications made to the basic elements of the curriculum, and the access elements, these transformations are defined at different levels as institutional, group and individual curricular project. Curricular adaptations are educational strategies to facilitate the teaching-learning process in students with disabilities, based on changes made to the regular curriculum to meet individual diversity without considering the origin of those differences: personal history, education, motivation and interest, pace and learning style. Inclusion aims to address diversity without discrimination, equal opportunity, its purpose is to eliminate the barrier of differences, talk about education for all, regardless of ethnicity, race,
\end{abstract}

\footnotetext{
${ }^{1}$ Universidad Educativa Patate, Tungurahua, Ecuador, morales2011@gmail.com

${ }^{2}$ Universidad Técnica de Ambato, Ambato, Ecuador le.velastegui@uta.edu.ec

${ }^{3}$ Escuela Pensionada la Merced Ambato, Ecuador, pablohomerovelastegui@ hotmail.com
} 
culture, religion, national origin, intellectual capacity, reflecting the right to a quality education for students with disabilities,

Keywords: Inclusion, curriculum adaptations, methodological strategies, diversity, disability.

CÓDIGO UNESCO: Educación 531204

\section{RESUMEN}

La defensa de los derechos humanos y de los valores democráticos de convivencia y el reconocimiento de la diversidad de los seres humanos y el respeto por sus diferencias, la búsqueda de la igualdad de oportunidades educativas para todas las personas la ayuda mutua, la cooperación y el intercambio de experiencias entre los países en el ámbito de la educación de personas con necesidades educativas especiales es la preocupación de los países latinoamericanos. La inclusión es un movimiento encaminado a transformar los sistemas educativos para atender a la diversidad de estudiantes es indispensable para hacer efectivo el derecho a la educación con equidad de oportunidades de acceso, permanencia y integración de todos los estudiantes, con énfasis en aquellos que están excluidos o en riesgo de ser marginados. La UNESCO realiza acciones de asesoramiento técnico, promoción de políticas y prácticas inclusivas; desarrollo y difusión de publicaciones, investigaciones y estudios de la educación inclusiva en la región, estas acciones favorecen cambios en las prácticas educativas, eliminando barreras desarrollando una educación de calidad para todos. Es un fenómeno mundial que pretende eliminar la relegación que tenían los individuos por sus necesidades físicas o intelectuales, en nuestro país se desarrollan estrategias para incluir en todos los ámbitos de la actividad humana a personas con discapacidad. Ecuador inicia con la inclusión como una política de estado poniendo énfasis en la inclusión educativa para atender la diversidad, buscando una educación con calidez y calidad, aspirando a que todos tengan una vida con dignidad, con igualdad de oportunidades, que conlleven al éxito de la inclusión de todos los estudiantes con diversos tipos de discapacidad, los procesos de inclusión son factores que despierten el respeto a la diversidad y reconocen los derechos constitucionales que deben cumplirse hacia las personas con discapacidad. Las adaptaciones curriculares son los ajustes o modificaciones que se realizan a los elementos básicos del currículo, y a los elementos de acceso, estas transformaciones se definen en diferentes niveles como proyecto curricular institucional, grupal e individualmente. Las adaptaciones curriculares son las estrategias educativas para facilitar el proceso de enseñanza aprendizaje en estudiantes con discapacidad, partiendo de modificaciones realizadas sobre el currículo ordinario, para atender a la diversidad individual sin considerar el origen de esas diferencias: historial personal, educativo, motivación e intereses, ritmo y estilo de aprendizaje. La inclusión tiene como finalidad atender a la diversidad sin discriminación, con igualdad de oportunidades, 
su fin es eliminar la barrera de las diferencias, hablar de educación para todos, sin distinción de etnia, raza, cultura, religión, origen, capacidad intelectual, plasmando el derecho a una educación de calidad, para estudiantes con capacidades diferentes,

Palabras clave: Inclusión, adaptaciones curriculares, estrategias metodológicas, diversidad, discapacidad.

\section{INTRODUCCIÓN}

La UNESCO define a la educación inclusiva como "El medio más efectivo para combatir las actitudes discriminatorias, creando comunidades de bienvenida, construyendo una sociedad inclusiva y alcanzando la educación para todos; además proporciona una educación eficaz para la mayoría de los niños, mejora la eficacia y, en último término la relación costeefectividad de todo sistema educativo" (UNESCO, 2008)

En la actualidad tanto políticas nacionales como colectivos sociales definen la idea de que los niños, niñas y adolescentes con necesidades educativas especiales (NEE), sean incluidos en las escuelas regulares, con el fin de ofrecer a estos estudiantes, la oportunidad de recibir una educación de calidad y calidez, así podrán aprovechar tanto los avances científicos como los beneficios legales que el estado ofrece, con un principio de igualdad para todos. Las instituciones educativas están invitados a llevar a cabo el proceso de inclusión a las personas con algún tipo de discapacidad; los docentes y todo el personal educativo deben estar atentos a que no exista ninguna actitud de discriminación, de la misma forma deben ayudar a que los sujetos incluidos se sientan seguros de sí mismo e integrarlos en todas las actividades que se desarrollan.

Podemos decir que en nuestro país Ecuador la inclusión de los adolescentes con NEE, al régimen educativo regular ha venido atravesando pequeños cambios, no solo desde lo estructural, sino también desde los aspectos culturales, sociales, científicos, filosóficos y legales; siendo la más fuerte la barrera actitudinal, por lo tanto las instituciones educativas deben estar preparadas para atender a estudiantes con NEE, sin ser excluidos por su condición social, cultural, ideología, sexo, etnia o alguna discapacidad física, intelectual, sensorial o sobre dotación intelectual; para esto deben sufrir profundas transformaciones, las cuales deben exigirse a las instituciones educativas regulares y adecuarse a sus diversos estilos, ritmos y formas diferentes de aprendizaje, sin sobre poner ningún tipo de barreras metodológicas o actitudinales, además se debe concebir a la escuela en un rol más enfocado al desarrollo de una cultura democrática, de tolerancia a lo diferente, en defensa de los derechos humanos y la equidad, al fomentar en los adolescentes la empatía hacía experiencias y sentimientos diferentes a los suyos.

Existen diferentes tipos de discapacidad que se generan por diversas causas, entre las cuales hemos de mencionar las siguientes: dotación intelectual, trastornos de comportamiento, discapacidad sensorial, visual, motora, intelectual, trastornos generalizados del desarrollo 
como el síndrome de asperger, el trastorno del espectro autista, síndrome de Down, las mismas que nos permitirán obtener un panorama más amplio en cuanto al área integral de trabajo del docente, quién deberá utilizar adecuadas estrategias metodológicas que se enfoquen al bienestar individual del estudiante. Las adaptaciones curriculares deben ser precisas para los estudiantes con NEE, de esta manera proporcionan ayuda y recursos que les faciliten avanzar en el logro de los aprendizajes establecidos, el currículo será abierto y flexible para mejorar la calidad de la enseñanza y asegurar la igualdad de oportunidades para todos.

\section{Problema o interrogantes}

- El problema identificado como la incorrecta aplicación de adaptaciones curriculares para los estudiantes con necesidades educativas específicas de educación inclusiva se halla revestido de varias causas, las mismas que priorizadas se circunscriben a tres esenciales, las que se indican a continuación.

- Requerimientos específicas de los estudiantes escasamente detectados, las herramientas curriculares actuales tienen una limitada incidencia en la detección de necesidades específicas de los estudiantes y tampoco permiten responder a ellas, para lograr el aprendizaje se requiere de estímulos, los mismos que deben tener validez pertinencia y finalidad; que se manifieste a través del rendimiento, permitiendo el cumplimiento de los objetivos educativos. Al no descubrir adecuadamente los requerimientos específicas de aquellos estudiantes se produce un desbalance en el aula donde no se consideran sus diferencias se utiliza la misma planificación que para el resto de alumnos esto provocara finalmente la deserción.

- La capacitación limitada sobre adaptaciones curriculares en la educación inclusiva, provoca que los docentes no se encuentren en capacidad de responder a los requerimientos del proceso de aprendizaje en la educación de estudiantes con necesidades específicas y apliquen un proceso estandarizado sin contemplar las diferencias individuales, transformado a la evaluación en un proceso sancionador que provoca temor en lugar de una herramienta para el aprendizaje.

- Planificación poco funcional para guiar la práctica docente, tiene a los profesionales de la educación sin herramientas para la innovación en la evaluación, para hacerla inclusiva y adaptada para los estudiantes con necesidades educativas específicas hace que los elementos del currículo se encuentre fuera de contexto y que el proceso no provoque un cambio interno en la conducta del estudiante que es el verdadero objetivo de la educación, por el contrario lo deja insatisfecho y le hace perder su autovaloración.

- ¿Las adaptaciones curriculares inciden en la inclusión de los estudiantes con Necesidades Educativas Específicas?

- ¿La Educación inclusiva permitirá desarrollar las destrezas de los estudiantes con 
Necesidades Educativas Específicas?

- ¿Qué herramientas se podrían utilizar para que el proceso de aprendizaje respondan a los estudiantes con Necesidades Educativas Específicas?

Se platea los siguientes objetivos: Determinar la incidencia de las Adaptaciones Curriculares aplicadas en la Unidad Educativa Benjamín Araujo para atender a los estudiantes con Necesidades Educativas Específicas.

Identificar el tipo de adaptaciones curriculares que se utilizan en la planificación para el aprendizaje de los estudiantes con Necesidades Educativas Específicas que se aplican dentro del Proceso de Enseñanza Aprendizaje

Analizar si la Educación Inclusiva permitirá desarrollar las destrezas de los estudiantes en los estudiantes con Necesidades Educativas Específicas.

Plantear modelos de planificación con adaptaciones curriculares para los diferentes grados de discapacidad intelectual, dentro del Proceso de Enseñanza Aprendizaje que serán aplicadas por los docentes en el aprendizaje de estudiantes con Necesidades Educativas Específicas en la Unidad Educativa Benjamín Araujo del cantón Patate.

\section{Metodología de análisis}

Está investigación se desarrollará bajo el enfoque del paradigma socio-crítico, buscando la participación de los diferentes actores, de carácter cuanti - cualitativo. Cuantitativo, porque se obtendrá información numérica y objetiva de las causas de la realidad actual, que será validada y sometida al análisis estadístico manteniendo la confiabilidad de los datos esto permitirá la comprobación de la hipótesis. Cualitativo, porque el ser humano es el sujeto y objeto de estudio, considerados como seres con cualidades, habilidades, destrezas, virtudes y debilidades diferentes; se propenderá a la comprensión de las causas y la definición de los hechos que se estudiarán, todo esto con un enfoque contextualizado que permitirá realizar un juicio de valor para diseñar una propuesta ejecutable.

Para desarrollar la presente investigación se utilizará la modalidad bibliográfica, mediante la utilización de libros, papers, leyes, reglamentos, acuerdos ministeriales para sustentar y profundizar los enfoques teóricos y conceptualizaciones de distintos autores

El Nivel o tipo de investigación es exploratoria porque es una metodología flexible, poco estructurada, para investigar el problema y conocer el contexto en el cual se desarrolla, permitiendo reconocer las variables de investigación, el propósito es explorar el problema de la evaluación de los estudiantes con discapacidad intelectual, permitiendo insertarse en la realidad de la evaluación aplicada en esta institución ir comprobando la hipótesis con la utilización de técnicas primarias como la observación, y la entrevista a personas involucradas para poder diseñar una posible solución al problema. 
La investigación será descriptiva porque permitirá identificar las características de los estudiantes con Necesidades Educativas Específicas y plantear sus relaciones con las estrategias de evaluación, conocer y establecer relaciones entre los factores, actores y variables del problema de investigación recopilación de la información a través de técnicas estructuradas como la encuesta

Identificando las causas y su relación con los efectos de la incorrecta aplicación de estrategias de evaluación para los estudiantes con discapacidad intelectual, se definirá y formulara las hipótesis, se elegirá las técnicas para la recolección y clasificación de datos Investigación de campo: La investigación se realizará en el mismo lugar en el que se producen los acontecimientos, donde se encuentra el objeto de estudio, porque permite que la investigación sea concebida como el análisis sistemático de problemas en la realidad recogiendo y registrando de manera secuencial los datos concernientes al problema propuesto para describirlos, interpretarlos, entender su naturaleza y factores de interés que fueron recogidos en forma directa de la realidad empleando como técnica la encuesta y como instrumento el cuestionario dirigido a docentes y estudiantes quienes proporcionaran información valiosa permitiendo descubrir, analizar e interpretar sus causas y efectos del problema objeto de la investigación.

El trabajo se basará en la investigación bibliográfica, mediante el uso de distintas fuentes como libros, revista, textos, papers, leyes, más documentos específicos sobre Educación y Evaluación Educativa y Necesidades Educativas Específicas con la intención de fortalecer la investigación explorando lo escrito en la comunidad científica.

Este tipo de investigación tiene como finalidad conocer el comportamiento y establecer el grado de relación de las variables de estudio, para ello se aplicará el estadígrafo denominado chi cuadrado, en este caso la aplicación de esta técnica permitirá al investigador formular hipótesis de investigación, en virtud de las necesidades que existen entre las variables tanto dependiente como independiente.

\section{Las adaptaciones curriculares}

Las adaptaciones curriculares se puede definir como los ajustes o modificaciones que se realizan a los elementos básicos del currículo, como también a los elementos de acceso, estas modificaciones se concretan en diferentes niveles como: en el proyecto curricular institucional, en el aula y a nivel individual.

Las adaptaciones curriculares son también una secuencia de acciones diseñadas para una población específica, con la intención de modificar uno o más elementos básicos del currículo 
con el fin de responder a las necesidades educativas de cada estudiante, en el contexto más normalizado posible.

Por otra parte las adaptaciones curriculares son estrategias educativas para facilitar el proceso de enseñanza-aprendizaje en algunos estudiantes con NEE. Estas estrategias pretenden, a partir de modificaciones más o menos extensas realizadas sobre el currículo ordinario, ser una respuesta a la diversidad individual independientemente del origen de esas diferencias: historial personal, historial educativo, motivación e intereses, ritmo y estilo de aprendizaje. La adaptación curricular es el ajuste o modificación que se realiza a los diferentes elementos de la oferta educativa común, para dar respuesta a los estudiantes con NEE, con o sin discapacidad.

\section{Consideraciones para realizar adaptaciones curriculares}

El punto de partida debe ser siempre el currículo ordinario, es necesario partir de una evaluación diagnóstica amplia del estudiante y una amplia contextualización de la enseñanzaaprendizaje, las adaptaciones deben realizarse con el menor grado posible de significatividad cambios que no afectan al currículo. Conseguir un objetivo en más o menos tiempo, introducir algunos contenidos, agrupar a los estudiantes de manera diferente.

Las adaptaciones deben tener en cuenta la realidad de los estudiantes y sus posibilidades de éxito, deben estar fundamentadas en dos principios: Principio de Normalización: Favorecer a los estudiantes con NEE, se beneficien siempre que sea posible, del mayor número de servicios educativos regulares, la vida de los estudiantes con NEE, debe ser la misma que la de cualquier otro en cuanto al ritmo, oportunidades y opciones.

Principio de Individualización: Intenta proporcionar a cada estudiante a partir de sus intereses, motivaciones y también en relación con sus capacidades, deficiencias y ritmos de aprendizaje- la respuesta educativa que necesite en cada momento para formarse como persona. (NIZBET, 2007). En el año 2011, La Ley Orgánica de Educación Intercultural (Ministerio de Educación, 2012), señala que los establecimientos educativos están obligados a recibir a todas las personas con discapacidad a crear los apoyos y adaptaciones físicas, curriculares y de promoción adecuadas a sus necesidades; y a procurar la capacitación del personal docente en las áreas de metodología y evaluación, específicas para la enseñanza de niños con capacidades para el proceso con interaprendizaje para una atención de calidad y calidez. Por lo tanto las adaptaciones curriculares responden a las necesidades de aprendizaje de cada estudiante, partiendo de la planificación y la práctica educativa cotidiana, por tanto son estrategias que facilitan el aprendizaje, no solo de las niñas/os y adolescentes con NEE, sino que también favorecen el proceso de aprendizaje de todos los y las estudiantes

\section{Las Adecuaciones de acceso al currículo}


Se puede decir que son las modificaciones o provisión de recursos espaciales, materiales, personales o de comunicación que van a facilitar que algunos estudiantes con NEE, puedan desarrollar el currículo ordinario o en su caso el currículo adaptado.

\section{Elementos personales y de organización}

Todas las personas involucradas en el proceso educativo deberán asumir una actitud positiva hacia los estudiantes con NEE. El equipo docente deberá poseer un nivel de formación suficiente y estar en constante capacitación para dar respuesta a las NEE. Deben tener capacidad para el trabajo en grupo

Una condición básica que debe darse en la organización de los elementos personales es la flexibilidad y el dinamismo. La organización de los elementos personales debe ser simple, clara, realista y coherente. Todos los profesionales deben participar en la toma de decisiones la organización de los elementos personales debe permitir el mayor grado posible de comunicación e interacción docente-estudiante y de los estudiantes entre sí. (Ministerio de Educación, 2012)

\section{Elementos materiales y su organización}

Organización del espacio

El mobiliario, equipamiento general de centro y recursos didácticos.

El tiempo y su organización Las condiciones que deben reunir cada uno de los elementos.

Entre las adecuaciones de acceso podemos distinguir las siguientes: En las instalaciones de los establecimientos educativos es necesario la colocación de rampas, barandales o señalizaciones en Braille, de tal manera que se permita el libre desplazamiento y acceso seguro de los niños, niñas y adolescentes con NEE, en todo el establecimiento. $\varpi$ Las relacionadas con cambios en el aula del estudiantado. Por ejemplo distribuir el mobiliario de manera distinta, elegir el aula más accesible para el niño, o colocar algunos materiales que ayuden a disminuir el nivel del ruido. Se busca que estas adecuaciones permitan compensar las dificultades del estudiante y promover su participación activa en la dinámica del trabajo del grupo. Las adecuaciones personales en adquisición de mobiliario para los estudiantes con discapacidad motora; el contar con materiales de apoyo, para los estudiantes con discapacidad visual; la adaptación de auxiliares auditivos o conseguir un tablero de comunicación o un intérprete de lenguaje manual para los alumnos con discapacidad auditiva. (NIZBET, 2007)

\section{Adaptaciones Curriculares Individualizadas}

Son todos aquellos ajustes o modificaciones que se efectúan en los diferentes elementos de la propuesta educativa desarrollada para un estudiante con el fin de responder a sus NEE y 
que no pueden ser compartidos por el resto de sus compañeros. Pueden ser de dos tipos: No Significativas: Modifican elementos no prescriptivos o básicos del currículo. Son adaptaciones en cuanto a los tiempos, las actividades, la metodología, las técnicas e instrumentos de evaluación en un momento determinado, cualquier estudiante tenga o no NEE, puede precisarlas es la estrategia fundamental para conseguir la individualización de la enseñanza y por tanto, tienen un carácter preventivo y compensador. Significativas o Muy Significativas: Modificaciones que se realizan desde la programación, previa evaluación psicopedagógica, y que afectan a los elementos prescriptivos del currículo oficial por modificar objetivos generales de la etapa, contenidos básicos y nucleares de las diferentes áreas curriculares y criterios de evaluación.

Estas adaptaciones pueden consistir en:

- Adecuar los objetivos, contenidos y criterios de evaluación.

- Priorizar determinados objetivos, contenidos y criterios de evaluación.

- Cambiar la temporalización de los objetivos y criterios de evaluación. Eliminar objetivos, contenidos y criterios de evaluación del nivel o ciclo correspondiente.

- Introducir contenidos, objetivos y criterios de evaluación de niveles o ciclos anteriores

\section{Adaptación curricular a los elementos básicos del currículo}

Se refiere al conjunto de modificaciones que se realizan en los contenidos, criterios y procedimientos evaluativos, actividades y metodologías para atender las diferencias individuales de los estudiantes con NEE, adaptaciones en los objetivos y contenidos.- Se refiere a las adaptaciones que se deben hacer al que, como y cuando enseñar, uno de los problemas fundamentales que se plantea en la escuela, es la determinación de los objetivos por conseguir, generalmente estos se han centrado en los resultados que los estudiantes debían lograr al final del proceso de enseñanza aprendizaje y en un campo de desarrollo muy concreto. Han sido precisamente los objetivos centrados en la adquisición de conocimientos iguales paras todos, los que han segregado a muchos estudiantes de la escuela ordinaria y por lo mismo hay que tener mayor cuidado de los conocimientos que deben ser desarrollados por los estudiantes y jóvenes con NEE.

\section{Las principales adaptaciones}

La priorización de determinados objetivos y contenidos secuenciación y temporalización. eliminación o inclusión de un objetivo o contenido de acuerdo a las necesidades específicas, incluir contenidos relativos a la adquisición de conceptos, procedimientos, valores, normas $\mathrm{y}$ actitudes

\section{Adaptaciones curriculares a las actividades}


Introducir actividades individuales y grupales, diseñar actividades amplias que tengan diferentes grados de dificultad y realización, proponer actividades diferentes para trabajar un mismo contenido, diseñar actividades que permitan diferentes posibilidades de ejecución y expresión, establecer momentos en los que confluyan diferentes actividades dentro del aula, realizar un diseño equilibrado del proceso de enseñanza-aprendizaje de gran grupo, pequeños grupos, trabajo en parejas e individual, planificar actividades de libre elección del estudiante, explicar claramente el tipo de actividades y como se van a realizar, planificar actividades en el entorno, que refuercen los conocimientos adquiridos Adaptaciones en la evaluación la evaluación es un elemento del currículo que, al igual que los otros susceptibles a ajustes, para atender a las NEE cuando se tiene estudiantes con dificultades se debe, establecer criterios de evaluación individualizados para ellos

La aplicación de criterios y estrategias diferenciales, establecer los criterios de promoción en función de las adaptaciones curriculares realizadas, utilizar técnicas, procedimientos e instrumentos de avaluación diferentes distintos a lo que se usa con el resto del grupo, tener en cuenta en la evaluación las ayudas pedagógicas concretas que el estudiante necesita, informar al estudiante de sus logros por pequeños que éstos sean, con el fin de motivarlos sicológica y técnicamente, aplicar a los estudiantes con NEE pruebas de evaluación adaptadas a sus propias necesidades, al mismo tiempo que sus compañeros.

Implicar en la evaluación, a las personas que han intervenido en el proceso de enseñanza aprendizaje. Adaptación de técnicas e instrumentos Evaluar en distintas situaciones de enseñanza - aprendizaje. Introducir el auto evaluación y la heteroevaluación en los estudiantes cuando sea posible.

\section{Estrategias de enseñanza para la inclusión}

Aprendizaje socio-emocional o en la escuela no solo se aprenden las competencias académicas; es un especio donde los niños aprenden a conocerse a sí mismos, vincularse con compañeros que son diferentes entre sí, relacionarse con figuras de autoridad distintas a sus padres y relacionarse con el mundo externo, todos estos vínculos debieran enriquecer su mundo emocional y ayudarlo a adquirir una identidad que le ayude a construir un proyecto de vida beneficioso para sí mismo y para los otros.

El paradigma del aprendizaje socioemocional puede constituir un modelo que favorezca los procesos de inclusión en el contexto escolar, entendiendo este como un proceso orientado a desarrollar competencias sociales y emocionales básicas, tales como la habilidad para reconocer y manejar emociones, el cuidado y la preocupación por los otros, tomar decisiones responsables, establecer relaciones positivas y enfrentar situaciones desafiantes de manera efectiva

La información aportada desde las neurociencias acerca de cómo las emociones afectan el aprendizaje cognitivo y socioemocional, ha abierto nuevos horizontes acerca de la 
transferencia de estos conocimientos hacia la enseñanza, centrándose en el desarrollo emocional positivo y en un enfoque más centrado en las competencias que tengan los estudiantes que en los déficit que puedan presentar, sin embargo, no parece haberse tomado suficientemente en cuenta en las acciones pedagógicas ni en la formación inicial de los profesores, constituyendo más bien una declaración de principios.

Las palabras que usamos para referirnos a los niños son esenciales para que construyan su concepto de sí mismos o su narrativa. Las miradas también influyen en esta construcción; hay miradas que manchan, que molestan, que ignoran, y hay otras que posibilitan y habilitan. Es preciso adoptar una mirada positiva centrada en las competencias y no en las dificultades, en el "yo posible" porque esto favorece el aprendizaje, la salud mental y el bienestar de los estudiantes.

Todos los estudiantes pueden aprender si reciben los apoyos necesarios, el aprendizaje social y emocional debe contemplarse en el currículo desde los primeros años, hay que ayudar a los niños a construir vínculos consigo mismos, con los otros y con la realidad. Existen cinco competencias básicas que es preciso desarrollar tanto en los docentes como en los estudiantes: la conciencia de sí mismo, la conciencia social, toma responsable de decisiones, autorregulación y manejo de relaciones.

Clima escolar positivo o Lo que se aprende en la escuela es lo que se construye en un contexto de las relaciones sociales profesor-estudiantes, entre estudiantes y entre los docentes. Para que un estudiante aprenda a entenderse positivamente debe observar y participar en comunidades en las que se establezcan relaciones positivas o el clima social escolar se refiere a la percepción que tienen los estudiantes de su contexto escolar y la percepción de los docentes de su entorno laboral, esta percepción incluye aspectos tales como las normas y creencias que caracterizan el clima escolar, el tipo de convivencia y las características de los vínculos existentes.

Las actitudes inclusivas de las diferencias, tanto de profesores como de estudiantes, son un factor que aporta significativamente a la creación de un buen clima escolar

El clima social escolar es uno de los determinantes más importantes en el éxito de la educación, tiene una mayor incidencia en el rendimiento escolar de los alumnos que los recursos materiales, la política escolar o los recursos personales la emoción abre o cierra las puertas al aprendizaje. Hay que crear climas en las aulas donde no haya tensión o temor, donde todos los niños se sientan seguros, acogidos y reconocidos.

Un clima emocional positivo se relaciona con la inteligencia emocional que tengan los miembros del grupo para resolver sus conflictos en forma pacífica cuando el clima emocional en que tiene lugar el aprendizaje se produce en una atmosfera positiva o nutritiva, la experiencia dejará una huella positiva en la memoria emocional esto se asocia a espacios 
educativos donde todos los miembros de la comunidad se sientan seguros, aceptados e incluidos. Por el contrario, los climas tóxicos generan interacciones agresivas y de maltrato, como se observa en las situaciones de acoso escolar.

Aprendizaje cooperativo o Las estructuras cooperativas son las más adecuadas para atender la diversidad; sin embargo en las aulas predominan las estructuras de aprendizaje individualistas y competitivas. o Inclusión y aprendizaje cooperativo son dos conceptos que se interrelacionan, solo pueden aprender juntos estudiantes diferentes en un contex to de cooperación en que todos se ayudan para alcanzar objetivos comunes, y a su vez, no puede haber un desarrollo pleno de la cooperación con el desarrollo de la solidaridad y el respecto a las diferencias que esta supone si previamente se han excluido de un aula a los que son diferentes.

El aprendizaje cooperativo se refiere al uso didáctico de equipos reducidos de estudiantes, de composición heterogénea en rendimiento y capacidad, aunque ocasionalmente pueden ser más homogéneos, utilizando una estructura de la actividad tal que asegure la participación equitativa de todos y se potencie al máximo la interacción simultánea entre ellos, con la finalidad de que todos los miembros de un equipo aprendan los contenidos escolares, cada uno hasta el máximo de sus posibilidades y aprendan, además, a trabajar en equipo el aprendizaje cooperativo promueve la participación activa de todos los estudiantes en el proceso de enseñanza y aprendizaje, acentuando su protagonismo y favorece la interacción entre pares. o La cooperación permite que el aula se convierta en una comunidad de aprendizaje, todos se benefician, aprenden más y se crea una interdependencia positiva entre los estudiantes y una identidad de equipo. o Las estrategias de aprendizaje cooperativo permiten que alumnos diferentes aprendan juntos. Se pueden trabajar los mismos contenidos persiguiendo diferentes objetivos o niveles de exigencia, o diversificando las actividades en función de las características de los estudiante en las estrategias de aprendizaje cooperativo se espera que cada estudiante aprenda no solo lo que el docente enseña, sino que contribuya a que lo aprendan sus compañeros y se beneficie de lo que ellos puedan aportarle, es una actividad que permite a los estudiantes contar unos con otros, colaborar y ayudarse mutuamente en el logro de sus aprendizajes cooperar no es lo mismo que colaborar.

La cooperación añade a la colaboración el desarrollo de la solidaridad, de la ayuda mutua que hace que los que en un principio simplemente colaboran para ser más eficaces acaben tejiendo entre ellos lazos afectivos más profundos que les permiten conseguir un objetivo común en las estructuras de aprendizaje cooperativo la evaluación siempre es individual, aunque se haya trabajado en equipo, y criterio, lo importante es que cada uno progrese en su aprendizaje en relación con su situación inicial y evaluar la capacidad de trabajar en equipo, formación docente para la inclusión

\section{La formación de los docentes}


Es un elemento imprescindible para el desarrollo de prácticas inclusivas, el aprendizaje cooperativo y el aprendizaje socio-emocional, sin embargo, el desarrollo personal y educación emocional de los docentes está insuficientemente desarrollado o definitivamente ausente en muchos programas de formación docente inicial y en servicio caracterizados por un marcado énfasis en lo cognitivo y en lo técnico, obviando lo emocional.

La relación que el profesor establece con sus estudiantes es fundamental en la generación de un clima saludable, tanto al interior de la sala de clases como en la escuelas, cuando los profesores son cálidos y apoyan a todos sus estudiantes se produce un sentido de apego y seguridad en el entorno escolar que les permite desarrollar plenamente sus potencialidades de aprendizaje, es difícil que un profesor que no tenga un adecuado desarrollo emocional, pueda convertirse en un modelo apropiado para los niños y favorecer su apertura a realidades diferentes, si los docentes no están abiertos a la diversidad no podrán desarrollar en los niños una actitud inclusiva frente a la emergencia de situaciones de violencia escolar, hostigamiento o intimidación se requiere que los docentes desarrollen competencias emocionales para manejar adecuadamente los conflictos, los docentes tienen dificultad para trabajar con estructuras de aprendizaje cooperativo, porque les cuesta entender que no todos los estudiantes tienen que trabajar lo mismo de forma simultánea, una modalidad muy eficaz para el cambio de actitudes y el fortalecimiento de capacidades de los docentes es la formación, a partir de la reflexión sobre las prácticas pedagógicas y la vivencia de las estrategias que tienen que aplicar luego en el aula en el caso del desarrollo socioemocional, una estrategia muy útil, tanto para la formación de los docentes como de los alumnos, es el uso de novelas, cuentos, películas, imágenes y metáforas, especialmente para promover cambios en las actitudes.

\section{Conclusiones.}

- Todo estudiante tiene derecho a educarse con sus pares aunque necesite intensos apoyos.

- La inclusión involucra un cambio de paradigma y una evolución profunda de los sistemas educativos la inclusión no es una simple inserción de los estudiantes a la oferta educativa disponible, es transformar el funcionamiento de las escuelas y avanzar hacia diseños completos de aprendizaje en los que el currículo y la enseñanza consideren las necesidades de aprendizaje de todos.

- En la inclusión el punto de atención es preparar a la escuela para que se adapte a los estudiantes en lugar de que estos se adapten a la escuela.

- La diversidad es parte de la naturaleza humana y se refiere a todos los estudiantes y no solo a ciertos grupos, los docentes no tenemos tiempo para conocer a los estudiantes y y mucho menos las excepciones, para terminar con esta breca aparece la inclusión

- La inclusión no es tanto un concepto como un modo de relación significa estar presente y participar, la inclusión depende del desarrollo de relaciones significativas y recíprocas ente los estudiantes. 
- Incluir no solo beneficia a quien es incluido sino todos los estudiantes quienes a través de la inclusión ven mejoran sus conductas emocionales y sociales. La verdadera inclusión depende del desarrollo de relaciones significativas entre los estudiantes.

- La inclusión permite un mayor desarrollo emocional de los estudiantes con el proceso de inclusión existen beneficios es posible observar una disminución de conductas agresivas y un aumento de conductas sociales.

- El hecho de convivir con personas de distintas etnias, capacidades, situaciones de vida género, culturas construye vínculos de apego con personas diferentes, lo que favorece una mentalidad abierta y aceptadora de las diferencias.

- La educación inclusiva sin distinción de ningún tipo debe acoger a todos los niños, niñas y adolescentes, y trabajar con las herramientas más oportunas para lograr un aprendizaje permanente y de calidad, así mismo debe contar con el compromiso de responsabilidad de toda la Comunidad Educativa.

- En los currículos educativos se incluyan acuerdos internacionales para que den resultados óptimos en este proceder educativo inclusivo; siendo por tanto necesaria la investigación, para ser aplicada en forma real y a sí mismo poner en ejecución los planes educativos que el gobierno ecuatoriano está desarrollando actualmente.

RECEIVED: JANUARY, 2017

REVISED: MARCH, 2017

\section{Referencias Bibliográficas}

- GARCÍA CEDILLO Ismael La Integración Educativa en el Aula Regular. Principios, Finalidades y Estrategias escuelas especiales de la Ciudad México España, 2012

- BÁRCENA, F. (2008). Aprendices del tiempo. La educación entre generaciones. Revista Todavía, Buenos Aires, núm. 21.

- DERRIDA, J. (2007). Aprender por fin a vivir. Buenos Aires: Amorrortu Editores

- CÓDIGO DE LA NIÑEZ Y LA ADOLESCENCIA. (2003). Quito.

- LOEI. (2012). Ley Orgánica de Educación Intercultural LOEI. QUITO: Editora Nacional. NIZBET, J. (2007). Estrategias del Aprendizaje. Madrid: Santillana. 


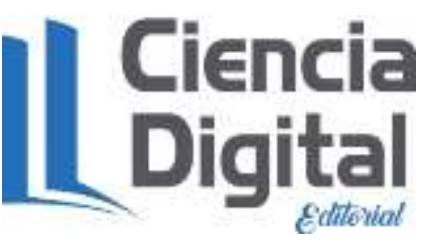

El artículo que se publica es de exclusiva responsabilidad de los autores y no necesariamente reflejan el pensamiento de la Revista Ciencia Digital.

El articulo queda en propiedad de la revista y, por tanto, su publicación parcial y/o total en otro medio tiene que ser autorizado por el director de la Revista Ciencia
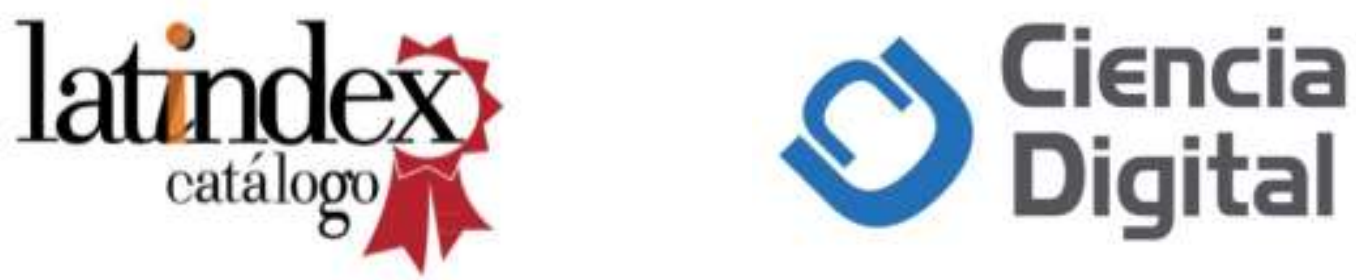